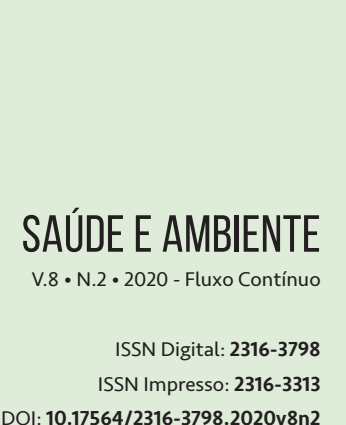

DOI: $10.17564 / 2316-3798.2020 v 8 n 2$

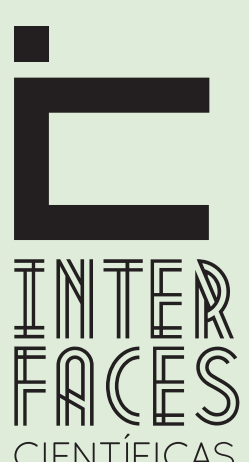

\section{ANÁLISE COMPARATIVA DE ADUBOS ORGÂNICOS ORIUNDOS DE DIFERENTES TIPOS DE COMPOSTAGEM}

COMPARATIVE ANALYSIS OF ORGANIC FERTILIZERS ORIUMS OF DIFFERENT COMPOUNDS

\section{ANÁLISIS COMPARATIVO DE FERTILIZANTES ORGÁNICOS ORIUNDOS DE DIFERENTES COMPUESTOS}

Sarah Samene Rocha Garcia ${ }^{1}$ Daniel Rocha Pereira ${ }^{2}$

Mikaelle Luzia Silva Dutra ${ }^{3}$

Alan Wesllen Pinto Ribeiro ${ }^{4}$ Keily Dammily Costa de Menezes ${ }^{5}$ Rafaelle Fonseca $\mathrm{Cruz}^{6}$

Rita de Cassia Mendonça Miranda ${ }^{7}$ Osman José de Aguiar Gerude Neto ${ }^{8}$

\section{RESUMO}

O objetivo deste trabalho foi analisar a qualidade do húmus produzido pela Perionyx excavatus com diferentes fontes de alimentos orgânicos, e comparando com o vermicomposto produzido em leiras de compostagem. 0 experimento foi executado em 60 dias, no Laboratório de Práticas Ambientais da UniCEUMA. Para as análises comparativas dos adubos, foram montados três tratamentos: T1- Adubo oriundo da Leira de copostagem ( $70 \%$ material fibroso, podas de gramas e folhas, e 30\% de resíduos orgânicos e fezes animal), T2- Adubo oriundo de resíduos organicos (100\% de resíduos organicos triturados para alimentação das minhocas); T3- Adubo organico para alimentação das minhocas oriundo das leiras em compostagem (30 dias de decoposição). Foram acondicionadas 20 minhocas por tratamento, as quais receberam a alimentação durante todo o período experimental. Ao final, os materiais de cada tratamento foram triturados, peneirados, pesados e acondicionados, lacrados e identificados, e encaminhadas para análises, nos laboratórios de Química e Física da UEMA, verificando-se as características físico - químicas. Os adubos analisados no experimento, todos apresentaram CTC acima de $70 \%$, indicando que são solos ricos em substâncias húmicas e com uma boa relação de $\mathrm{C} / \mathrm{N}$. As minhocas Violeta do Himalaia, deram uma resposta qualitativa superior quanto a produção de húmus, quando alimentadas com material oriundo da leira, tendendo se a acreditar, que o processo de compostagem mista (leira + minhocas) seja a melhor forma de produção de vermicomposto orgânico.

\section{PALAVRAS-CHAVE}

Violeta Himalaia, Húmus, Vermicomposto. 


\section{ABSTRACT}

The objective of this work was to analyze the quality of the humus produced by Perionyx excavatus with different sources of organic food, and comparing it with the vermicompost produced in compost beds. The experiment was carried out in 60 days, at the Laboratory of Environmental Practices of UniCEUMA. For the comparative analyzes of the fertilizers, three treatments were set up: T1- Fertilizer from the Leita of coping ( $70 \%$ fibrous material, pruning of grams and leaves, and $30 \%$ of organic residues and animal faeces), T2 - Fertilizer from organic residues (100\% crushed organic waste to feed earthworms); T3 - Organic fertilizer to feed the worms from the composting line (30 days of decoposition). Twenty worms were conditioned by treatment, which received the feeding during the whole experimental period. At the end, the materials of each treatment were crushed, sieved, weighed and conditioned, sealed and identified, and sent for analysis in the Chemistry and Physics laboratories of the UEMA, to verify the physicochemical characteristics. The fertilizers analyzed in the experiment all presented CTC above $70 \%$, indicating that they are soils rich in humic substances and with a good C / $\mathrm{N}$ ratio. The Himalayan Violet earthworms gave a superior qualitative response to humus production, when fed with material from the litter, tending to believe that the mixed composting process (Leira + earthworms) is the best way to produce vermicompost organic.

\section{KEYWORDS}

Violet Himalayan, Humus, Vermicompost.

\section{RESUMEN}

El objetivo de este trabajo fue analizar la calidad del humus producido por la Perionyx excavatus con diferentes fuentes de alimentos orgánicos, y comparando con el vermicompost producido en hileras de compostaje. El experimento fue ejecutado en 60 días, en el Laboratorio de Prácticas Ambientales de la UniCEUMA. Para el análisis comparativo de los abonos, fueron montados tres tratamientos: T1- Abono oriundo de la hilera de compostaje (70\% material fibroso, podas de gramos y hojas, y $30 \%$ de residuos orgánicos y heces animales), T2 - Abono procedente de residuos orgánicos (100\% de residuos orgánicos triturados para la alimentación de las lombrices); - T3 - Abono orgánico para la alimentación de las lombrices de las leñas en compostaje (30 días de descomposición). Se acondicionaron 20 lombrices por tratamiento, las cuales, recibieron la alimentación durante todo el período experimental. Al final, los materiales de cada tratamiento fueron triturados, tamizados, pesados y acondicionados, sellados e identificados, y encaminados para análisis, en los laboratorios de Química y Física de la UEMA, verificándose las características físico - químicas. Los abonos analizados en el 
experimento, todos presentaron CTC por encima del 70\%, indicando que son suelos ricos en sustancias húmedas y con una buena relación de C / N. Las lombrices Violeta del Himalaya, dieron una respuesta cualitativa superior en cuanto a la producción de humus, cuando se alimentan con material oriundo de la leña, tendiéndose a creer, que el proceso de compostaje mixto (leña + lombrices) sea la mejor forma de producción de vermicompost orgánico.

\section{PALABRAS CLAVE}

Violeta del Himalaya, Humus, Vermicompost.

\section{INTRODUCÇ̃̃O}

A minhoca Perionyx excavatus (PERRIER, 1872) (Annelida, Oligochaeta), conhecida como violeta-do-himalaia, serve para otimizar o processo de tratamento do resíduo orgânico, atuando com grande capacidade na transformação do resíduo em matéria orgânica. Segundo Guimarães (2016), esta é a espécie melhor indicada para povoar os minhocários de regiões mais quentes: a eficiência de humificação (produção de húmus) e os índices reprodutivos sob o calor rigoroso são extraordinariamente expressivos.

A Perionyx excavatus (violeta do Himalaia) é mais inquieta que outras espécies. Seu corpo apresenta tom violeta, excepcionalmente quando exposta à luz solar. Produz de 1,2 a 2,7 casulos por dia, porém de cada um destes, originam-se de 1,0 a 1,1 minhocas, com período de incubação em torno de 18 dias, atingindo a maturidade sexual após 28 a 42 dias. Essa espécie se desenvolve adequadamente em temperaturas entre $25^{\circ} \mathrm{C}$ a $37^{\circ} \mathrm{C}$ (EDWARDS; LOFTY, 1977).

Há uma série de benefícios em produzir e utilizar o húmus de minhoca ou vermicomposto. De uma forma geral, é possível fazer húmus de qualquer material orgânico que se decomponha; logo, qualquer resíduo orgânico disponível e não aproveitado na propriedade rural pode ser utilizado. O húmus de minhoca, que nada mais é do que as excreções da minhoca, quando aplicado ao solo, atua de forma benéfica sobre suas características físicas, químicas e biológicas, favorecendo a sua conservação e auxiliando o desenvolvimento das plantas (SCHIEDECK et al., 2010).

Segundo Baldotto e colaboradores (2013), o húmus é constituído de substâncias húmicas (SH), que são misturas heterogênicas de compostos orgânicos unidos por interações intermoleculares fracas de caráter hidrofóbico e por ligações de hidrogênio. São fracionadas em ácidos húmicos (AHs), ácidos fúlvicos (AFs) e huminas (HUM).

“A vermicompostagem é o processo de transformação de matéria orgânica recente, isto é, pouco degradada, por meio da ação das minhocas junto com a flora que vive em seu trato digestivo, em matéria orgânica estabilizada" (DORES-SILVA et al., 2011, p. 956). A vermicompostagem é um sistema tecnológico reconhecido como de baixo custo, o qual pode ter alto valor nutricional para as plantas 
cujo efeito se dá em curto espaço de tempo, reduzindo substancialmente o tempo de percurso entre a fotossíntese e o húmus (GARG; YADAY, 2011; MELO JÚNIOR et al., 2012; BASSACO et al., 2015).

De acordo com Albanell e colaboradores (1988), o processo de vermicompostagem é o resultado da combinação da ação de minhocas e dos microorganismos que habitam seus intestinos e finalmente dar origem ao vermicomposto. Assim o objetivo deste trabalho é analisar a qualidade do húmus produzido pela Perionyx excavatus com diferentes fontes de alimentos orgânicos e comparando com o vermicomposto produzido em leiras de compostagem.

\section{MATERIAIS E MÉTODOS}

Esta pesquisa foi realizada com abordagem de natureza aplicada, quanto a forma de abordagem qualitativa e aos seus objetivos exploratória e com os procedimento de caráter experimental.

Os tratamentos são compostos da seguinte forma (TABELA 1):

T1 - Adubo oriundo da Leira de copostagem (70\% material fibroso, podas de gramas e folhas de árvores, $15 \%$ de resíduos orgânicos e $15 \%$ de fezes animal), grande parte destes ingredientes coletados na própria Universidade, exceto o esterco bovino, que fora coletado na Universidade Estadual do Maranhão (UEMA).

T2 - Adubo oriundo de resíduos orgânicos (neste tratamento foi fornecido para as minhocas Violeta do Himalaia (Perionyx excavatus) apenas resíduos orgânicos triturados);

T3 - Adubo orgânico, oriundo da alimentação das minhocas com a leira em decomposição (as minhocas foram alimentadas commaterial da leira de compostagem com 30 dias de decoposição).

Tabela 1 - Composição dos tratamentos experimentais

\begin{tabular}{|c|c|c|c|}
\hline Tratamento & $\begin{array}{c}\text { Material Fibroso } \\
\text { (\%) }\end{array}$ & $\begin{array}{c}\text { Fezes Animal } \\
(\mathbf{\%})\end{array}$ & $\begin{array}{c}\text { Material Orgânico } \\
\text { (\%) }\end{array}$ \\
\hline $\mathrm{T} 1$ & 70 & 15 & 15 \\
\hline $\mathrm{T} 2$ & 0 & 0 & 100 \\
\hline $\mathrm{T} 3$ & 70 & 15 & 15 \\
\hline
\end{tabular}

Fonte: Dados da pesquisa

Na produção dos adubos orgânicos utilizou-se as minhocas da espécie Violeta do Himalaia (Perionyx excavatus) que facilmente adaptam-se a variados climas, segundo Gonzaga (2018), pois apresentam alta capacidade de produção de húmus e de reprodução, nas condições climáticas de São Luís-MA. As Perionyx excavatus foram alimentadas com dois tipos diferentes de resíduos orgânicos, 
como descritos acima. As minhocas foram adquiridas do site da empresa Minhobox ${ }^{\oplus}$, sendo que 0 acondicionamento delas se deu por meio de dois recipientes de polímero utilizados como composteiras (T2 e T3), sendo acondicionadas 20 minhocas por recipiente, as quais receberam a alimentação durante todo o período experimental.

O experimento teve duração de 60 dias e ao final os materiais de cada tratamento foram peneirados, pesados, acondicionados, lacrados, identificados, encaminhados para análises, nos laboratórios de Química e Física dos Solos da Universidade Estadual do Maranhão (UEMA), verificando-se as características químicas ( $\mathrm{pH}$, matéria orgânica, fósforo, potássio, sódio, cálcio, alumínio e manganês) e físicas (densidade do solo, densidade das partículas, granulometria e porosidade) de acordo com os métodos da Embrapa (2017).

As amostras foram levadas a estufa para processo inicial do procedimento, que é a secagem, utilizando o método terra fina seca pelo ar (TFSA), após foram passadas por uma peneira de $2 \mathrm{~mm}$ onde foram cachimbadas, para assim, haver os ataques com soluções para avaliar o teor total de cada parâmetro.

\subsection{ANÁLISES QUUIMICAS}

\subsubsection{PH}

Para a análise do $\mathrm{pH}$, foram adicionados $25 \mathrm{ml}$ de $\mathrm{CaCl}_{2}$ (cloreto de cálcio) com $10 \mathrm{~g}$ de solo, após foi agitada em mesa agitadora, em seguida executada a leitura no pHmetro através de eletrodos mergulhados na amostra.

\subsubsection{MATÉRIA ORGÂNICA}

Agitou-se por 10 minutos a matéria orgânica, adicionando-a em solução sulfocrômica, retirando o sobrenadante e analisando o material no espectrofotômetro com o comprimento de onda de $650 \mathrm{~nm}$.

\subsubsection{SÓDIO E POTÁSSIO}

Para determinar a quantidade de sódio e potássio, foi feito o ataque das amostras, foi adicionado $25 \mathrm{ml}$ da solução de Mehlich, de onde é retirado $5 \mathrm{ml}$ de sobrenadante e em seguida, feito a leitura pelo fotômetro.

\subsubsection{FÓSFORO}

Para análise do fósforo, foi pesado 2,5g de solo e adicionado $100 \mathrm{ml}$ da solução de Mehlich, $10 \mathrm{ml}$ de molibdato de amônio e 30mg de ácido ascórbico, e assim feito sua leitura no espectrofotômetro, com o comprimento de onda de $660 \mathrm{~nm}$.

\subsubsection{CÁLCIO, ALUMÍNIO E MAGNÉSIO}

A análise do cálcio, alumínio e magnésio, ocorreu por meio da extração da solução $K C I 1 N$, misturada ácido etilenodiaminotetracético (EDTA) 0,025 mol L-1, originalmente na cor avermelhada e depois de adicionada se torna azul, obtendo assim o resultado pelo uso do espectrofotômetro. 


\subsection{ANÁLISES FÍSICAS}

\subsubsection{DENSIDADE DO SOLO}

A densidade é calculada por meio da relação entre a massa da amostra do solo seco e da soma do volume das partículas e dos poros, onde são colocados em anéis volumétricos e assim pesando a amostra e calculado por meio da equação a seguir:

$$
\text { Ds }=\frac{\text { Massa }}{\text { Volume }}
$$

\subsubsection{DENSIDADE DAS PARTÍCULAS OU POROSIDADE}

A densidade das partículas é calculada por meio da relação entre a massa da amostra do solo e o volume ocupado pelas amostras, na qual a massa é obtida por pesagem em balança analítica e o volume com auxílio de balão volumétrico, é adicionado álcool etílico para a determinação do volume do liquido deslocado pela massa do solo.

$$
P s=\frac{\text { Massa do solo }}{\text { Volume do solo }}
$$

\subsubsection{GRANULOMETRIA}

A granulometria corresponde a distribuição e do tamanho das partículas que constitui o solo, também chamadas de frações granulométricas, que são divididas em três tipos: areia, silte e argila. A análise é feita pela pesagem de $20 \mathrm{~g}$ de solo (TFSA), adiciona $100 \mathrm{ml}$ de água deionizada, $10 \mathrm{ml}$ de hidróxido de sódio e $10 \mathrm{ml}$ de hexametafosfato de sódio, após agitada, passada por peneira. A mesma é levada para a estufa para que haja a evaporação, em seguida é levada ao dessecador, e assim, é feita a determinação das frações do solo.

\section{RESULTADOS}

Nas analises químicas (TABELA 2), observou-se que no T3 a maior quantidade de Fósforo (P) em relação aos demais tratamentos, demonstrando que este possui uma capacidade de fertilização superior, pois este elementoe essencial para o crescimento e desenvolvimento das plantas. 0 tratamanto em questão tambem apresentou indicies superiores de Ca disponibilizados no solo.

$\mathrm{O} \mathrm{K}$ apresentou maiores índices no T2, onde este serve para sustentar o crescimento da planta. Plantas com deficiência de potássio são menos resistentes a estiagem. Uma planta suprida por potássio é mais resistente ao ataque de pragas e doenças. O Al apresntou níveis altos no T3 (16 mmol/ $\mathrm{dm}^{3}$ ), mais esta concentração acaba sendo neutralizada pela alta concentração de matéria orgânica desse tratamento que apresentou $214 \mathrm{~g} / \mathrm{dm}^{3}$. 
A capacidade de troca catiônica (CTC) pode ser obtida por soma de bases, conforme a fórmula: $\mathrm{CTC}=\mathrm{Ca}^{2+}+\mathrm{Mg}^{2+}+\mathrm{K}^{+}+\mathrm{Na}^{+}+\mathrm{H}+\mathrm{Al}$. A relação CTC/COT é um indicativo do grau de maturação e qualidade dos fertilizantes orgânicos e é considerada como mais apropriada que a relação $\mathrm{C} / \mathrm{N}$ para indicar o grau de humificação de materiais orgânicos, pois esta última pode ser afetada seriamente pela presença de nitrogênio amoniacal, como ocorre no esterco de galinha. Os tratamentos analisados no experimento apresentaram CTC acima de 70\% (T1 - 86,8\%; T2 - 96,5\% e T3 - 94,1\%), indicando que são solos ricos em substâncias húmicas e com uma boa relação de $\mathrm{C} / \mathrm{N}$.

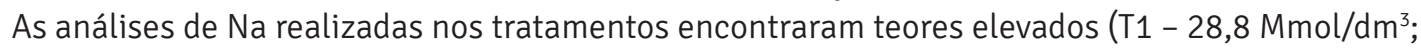

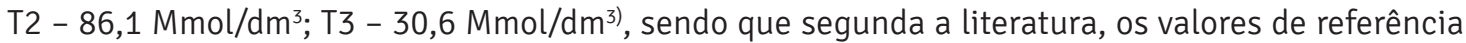
indicados para melhor desempenho do solo se encontram entre $5,0 \mathrm{Mmol} / \mathrm{dm}^{3}$ e $8,0 \mathrm{Mmol} / \mathrm{dm}^{3}$, o que faz com que esses resultados indiquem um alto teor salino (EMBRAPA, 2015).

É necessário ressaltar que todos os tratamentos encontram-se altos índices de MO, pois os mesmos indicam por meio da análise feitas, que os valores estão acima de $60 \mathrm{~g} / \mathrm{dm}^{3}$, sendo este considerado ideal para a literatura $\mathrm{T}\left(1-94 \mathrm{~g} / \mathrm{dm}^{3}, \mathrm{~T} 2-71 \mathrm{~g} / \mathrm{dm}^{3}, \mathrm{~T} 3-214 \mathrm{~g} / \mathrm{dm}^{3}\right)$, indicando que quanto maior o teor de MO, melhor a qualidade do composto orgânico e maior a disponibilidade de nutrientes tanto para o solo, quanto para as culturas.

De acordo com as análises físicas dos tratamentos (TABELA 3), o T1 - caracterizou-se como um solo de textura Areia Franca, pois ele dispõe de baixos indices de silte (11\%) e elevada quantidade de areia fina e gossa 28 e 53\% respectivamente. Os tratametos T2 e T3 apresentaram uma grande quantidade de silte em sua composição, por isso sendo classificados como Franco Siltoso, este tipo de solo tende a reter mais os nutrientes em suas camadas superiores, pois tem uma poridade um pouco mais reduzida, mas ainda se apresentando como bom dispositor de nutrientes para as plantas. 


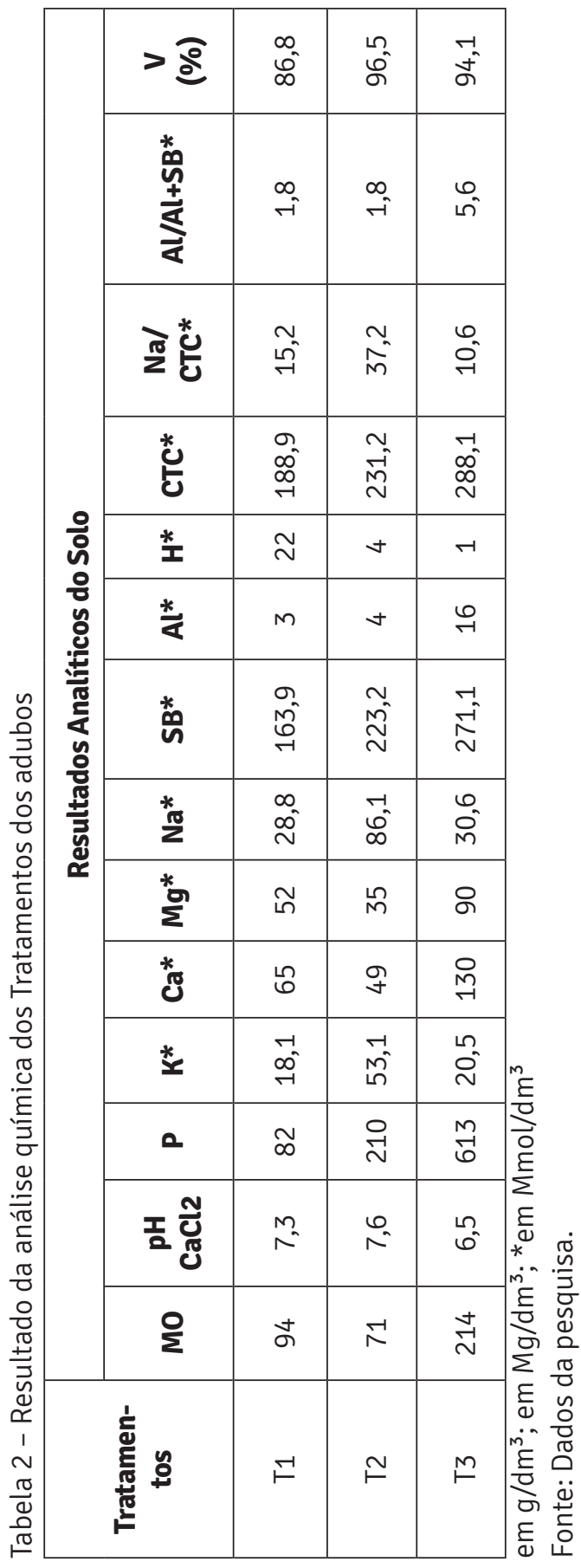

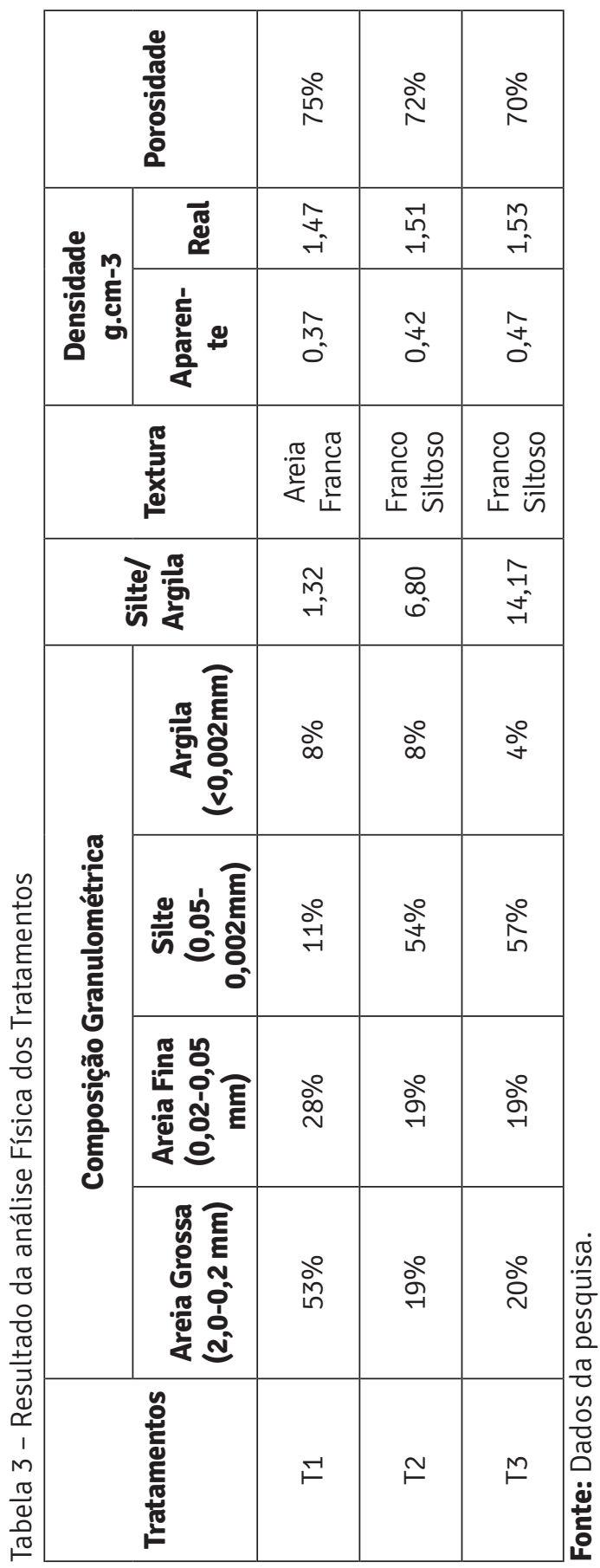




\section{DISCUSSÃO}

Solos com características de textura de Areia Franca, são compostos por macro e micro poros, facilitando assim a disposição de seus nutrientes (WEIL et al., 2016). Os siltoarenosos mostram uma seleção menor, ou seja, mais heterogeneidade na granulometria, isso reduz a permeabilidade do material (REINERT; REICHERT, 2006).

Segundo Giracca (2017), o fósforo é o macronutriente, que é absorvido em menores quantidades em relação aos outros, contudo a presença do mesmo no solo é indispensável para o crescimento e produção vegetal. Este contribue diretamente com o crescimento prematuro das raízes, qualidade de frutas, verduras, grãos e formação das sementes. Já o Ca desempenha um papel de suma importância, pois este é responsável por reduzir a acidez do solo, melhorar o crescimento das raízes e aumentar a atividade microbiana.

Segundo Nascimento e colaboradores (2015), com a adição de verme compsoto na nutrição dos vegetais, temos uma fonte de K que está constantemente disponibilizando este elemento no solo e pra as plantas.

Conforme Miguel e colaboradores (2010), o alumínio, dependendo de sua quantidade, vem sendo um vilão para a agricultura, pois seus níveis de toxidade fazem com que seja reduzido o crescimento radicular de plantas frágeis, sendo que isso afeta diretamente no alongamento e divisão celular, o que tem como consequência a ineficiência da planta com seu raizamento superficial, em obter água e nutrientes do subsolo de forma adequada, dessa forma, ficando menos resistentes a estiagem e menos produtiva.

Pode-se afirmar que a saturação por bases é a proporção da capacidade de troca catiônica ocupada pelas bases. Solos com saturação por bases maiores que $70 \%$ indicam que não há necessidade de calagem. Segundo Sobral e colaboradores (2015), para a correção de solos, estes devem possuir saturação por base menor que $50 \%$, pois possuem cargas ocupadas por componentes de acidez ( $\mathrm{H}$ ou $\mathrm{Al}$ ).

De acordo com Dores-Silva e colaboradores (2011), todos os diferentes parâmetros de caracterização de vermicomposto estão correlacionados em maior ou menor grau, pois refletem basicamente o mesmo processo de reações de estabilização de compostos orgânicos. A vantagem da utilização da capacidade de troca de cátions é que, além de qualificar o material orgânico, esse índice traz uma informação muito significativa do ponto de vista agronômico, ou seja, a melhoria de retenção de nutrientes que esses produtos podem proporcionar ao serem incorporados ao solo.

\section{CONCLUSÃO}

Os tratamentos analisados apresentaram índices físico-químicos acima nos descritos na literatura para solos saudáveis. Isso demonstra que independente da forma de compostagem (com ou sem minhocas) os adubos orgânicos gerados têm potencial elevado de liberação de nutrientes para o solo.

As minhocas Violeta do Himalaia, deram uma resposta qualitativa superior quanto a produção de húmus, quando alimentadas com material oriundo da leira de compostagem. E isso, leva-se a 
crer, que o processo de compostagem mista (leira + minhocas) seja a melhor forma de produzir um adubo orgânico mais rico em nutrientes, com maior capacidade de disponibilização tanto para o solo, quanto para as plantas.

\section{REFERÊNCIAS}

ALBANELL, E. et al. Chemical changes during vermicomposting (Eisenia fetida) of sheep manure mixed with cotton industrial wastes. Biol. Fertility Soils, v. 6, p. 266-269, 1988.

BALDOTTO, M. A. et al. Frações da matéria orgânica e propriedades redox de substâncias húmicas em sedimentos de oceanos profundos. Quím. Nova, v. 36, n. 9, p.1288-1295, 2013.

BASSACO, A. C. et al. Caracterização química de resíduos de origem animal e comportamento de Eisenia Andrei. Ciên. Natura, v. 37, n. 1, p. 45-51, 2015.

DORES-SILVA, P. R. et al. Acompanhamento químico da vermicompostagem de lodo de esgoto doméstico. Quím. Nova, v. 34, n. 6, p. 956-961, 2011.

EDWARDS, C. A.; LOFTY, J. F. Biology of earthworms. $2^{\text {nd }}$. ed. London: Chapman e Hall, 1977.

GARG, V. K.; YADAY, A. Vermicomposting: an effective tool for the management of invasive weed Parthenium hysterophorus. Bioresource Technol., v.102, n.10, p. 5891-5895, 2011.

GIRACCA, E. Fertilizantes: Nutrientes. Disponível em: www.agrolink.com.br /fertilizantes. Acesso em: 9 jun. 2018.

GONZAGA, L. F. Avaliação da qualidade do húmus produzido pela espécie de minhoca violeta do himalaia (Perionyx excavatus), produzidos nas condições climáticas de São Luís - MA. XI Seminário de Iniciação Científica, 11, 2018. Anais..., São Luís-MA, 2018.

GUIMARÃES, A. A. Reciclagem de resíduos orgânicos através da minhocultura. In: RESENDE, L.O. et al. (Org.). Sustentabilidade: Tópicos da Zona da Mata Mineira. Juiz de Fora: Real, 2016.

LOUREIRO, D. C. et al. Compostagem e vermicompostagem de resíduos domiciliares com esterco bovino para a produção de insumo orgânico. Pesq. Agropec. Bras., v. 42, n. 7, p. 1043-1048, 2007.

MELO JÚNIOR, H. B. et al. Efeito da ação decompositora da minhoca californiana (Lumbricus rubellus) na composição química de um fertilizante organomineral. Biosciense J., v. 28, suppl. 1, p. 170-178, 2012. 
MIGUEL, P. et al. Efeitos tóxicos do alumínio no crescimento das plantas: mecanismos de tolerância, sintomas, efeitos fisiológicos, bioquímicos e controles genéticos. CES Rev., v. 24, n. 1, p. 13-32, 2010.

NASCIMENTO, A. et al. Caracterização de vermicomposto produzido com palha de café e esterco bovino. Rev. Caatinga, v. 28, n. 4, p. 1-9, 2015.

REINERT, D. J.; REICHERT, J. M. Propriedades físicas do solo. Santa Maria: UFSM, 2006.

SCHIEDECK, G. et al. Aspectos culturais associados às minhocas no Brasil. Acta Zool. Mex., v. 26, n. 2, p. 19-33, 2010. 
1 Graduada em Engenharia Ambiental pela Universidade CEUMA. E-mail: sarahsamenny@hotmail.com

2 Mestre em Energia e Ambiente pela Universidade Federal do Maranhão - UFMA; Graduado em Engenharia Ambiental, Universidade CEUMA; Professor do curso de Engenharia Ambiental da Universidade CEUMA.

E-mail: daniel.rocha.drp@gmail.com

3 Acadêmica de Engenharia Ambiental, Universidade CEUMA. E-mail: mickaelle.silva@live.com

4 Graduado em Engenharia Ambiental, Universidade CEUMA. E-mail: alanfera71@hotmail.com

5 Graduada em Engenharia Ambiental, Universidade CEUMA. E-mail: dammymenezes10@gmail.com

6 Graduada em Engenharia Ambiental, Universidade CEUMA. E-mail: rafaellefonseca.c@hotmail.com

7 Doutora em Biologia dos Fungos e Graduada em Ciências Biológicas, Universidade Federal do Pernambuco - UFPE; Professora do curso de Engenharia Ambiental da Universidade CEUMA. E-mail: rita.miranda@ceuma.br

8 Mestre em Ciência Animal, pela Universidade Federal do Maranhão - UFMA; Graduado em Zootecnia pela Universidade Estadual do Maranhão - UEMA; Professor do curso de Engenharia Ambiental da Universidade CEUMA.

E-mail: osman.aguiar@ceuma.br

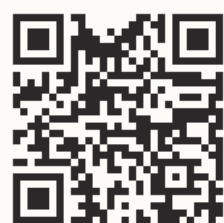

A autenticidade desse artigo pode ser conferida no site https://periodicos. set.edu.br

\section{Como citar este artigo:}

ROMEO, Andrea. Lo special account del fenomeno religioso nel dibattito nordamericano. Argumenta Journal Law, Jacarezinho - PR, Brasil, n. 29., 2018, p. 15-48. DOI: $10.17564 / 2316-3828.2018 v 7 n 1 p 13-24$

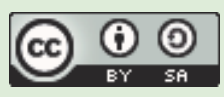

Este artigo é licenciado na modalidade acesso abertosob a Atribuição-Compartilhalgual CC BY-SA

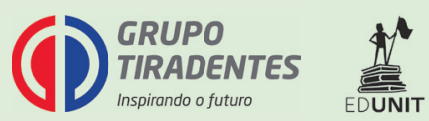

